\title{
Imaging of internal cracks in concrete structures using the surface rendering technique
}

\author{
Po-Liang Yeh, Pei-Ling Liu* \\ Institute of Applied Mechanics, National Taiwan University, Taipei 106, Taiwan
}

\section{A R T I C L E I N F O}

Article history:

Received 17 October 2007

Received in revised form

20 March 2008

Accepted 21 May 2008

\section{Keywords:}

Concrete

Internal crack

Imaging

Surface rendering method

Nondestructive test

\begin{abstract}
A B S T R A C T
The impact echo method is effective in the inspection of concrete defects. If the test area is large and many tests are performed, it is difficult to get a picture of the concrete interior by examining a series of test spectra. In order to provide the engineers with a more direct way of detecting the defects in the structure, this study proposes a three-dimensional (3D) imaging method to depict the internal cracks in concrete structures. To acquire the test data, a mesh is drawn on the surface of the concrete. Then, impact echo tests are performed at the grids. The recorded signals are processed to obtain the depth spectra of the concrete. Finally, the surface rendering technique is adopted to construct the 3D image of the concrete interior. Both numerical simulations and model tests are used to verify the proposed imaging method. It is seen that surface rendering technique can be used to show the internal cracks in the concrete specimens successfully.
\end{abstract}

(c) 2008 Elsevier Ltd. All rights reserved.

\section{Introduction}

Concrete is a commonly used construction material for structures because it is economical and durable. However, the existence of defect may decrease the strength of the structure and induce failure of the structure. Hence, several non-destructive testing methods were developed to evaluate the safety of concrete structures. Among these methods, the impact echo test is the most widely used technique to detect cracks in concrete structures.

The impact echo method was developed in 1980s [1]. In the impact echo test, an impact is applied to the surface of the target structure. Then, the impact response is measured and transformed to the frequency domain by the Fourier transform (FT). Since interfaces, cracks, or voids induce echo of the stress waves, peaks will form in the Fourier spectrum. As such, the spectrum will reveal the size of the structure or the location of the defect. The echo frequency $f$ and the depth of the interface, $d$, are related by the following formula:

$d=\frac{C_{\mathrm{p}}}{2 f}$

where $C_{\mathrm{p}}$ is the velocity of the longitudinal wave. Therefore, by locating the peaks in the Fourier spectrum, one can determine the depth of the structure or the internal cracks.

\footnotetext{
* Corresponding author.

E-mail address: peiling@iam.ntu.edu.tw (P.-L. Liu).
}

The Fourier spectrum is useful in the detection of interfaces. However, the FT is built on the assumption of a stationary signal. Unfortunately, the impact response of a structure is not stationary, and its frequency content varies with time. Hence, ripples and multiple peaks may appear in the spectrum and make the interpretation difficult. Therefore, time-frequency analysis has been proposed in the literature as an alternative technique to process impact echo signals [2-7].

According to Yeh and Liu [7], the wavelet transform is an effective time-frequency analysis that may generate a smooth spectrum. However, it never surpasses FT in frequency resolution due to the uncertainty principle. The echo peak in the marginal spectrum of the wavelet transform is not as sharp as in the Fourier spectrum. In order to incorporate the advantages of both Fourier and wavelet transforms, Yeh and Liu [7] proposed the enhanced Fourier spectrum as follows:

$E(f)=X(f) Y(f)$

where $X(f)$ is the Fourier spectrum, $Y(f)$ is the wavelet marginal spectrum, and $E(f)$ is the enhanced Fourier spectrum.

The wavelet marginal spectrum can be considered as a bandpass filter. Multiplying $X(f)$ with $Y(f)$ amounts to applying the band-pass filter $Y(f)$ to $X(f)$. The frequency components in $X(f)$ around the peak of $Y(f)$ are magnified, while the frequency components away from the peak are held back. As such, the sharpness of the echo peaks can be retained while suppressing the unwanted ripples and multiple peaks in the Fourier spectrum.

The impact echo test is a point-wise detection method. If the test area is large, a vast amount of test points are needed. It makes 
the interpretation of test results complicated and time-consuming. For a rapid and effective diagnosis, several two-dimensional (2D) imaging methods were proposed to detect the internal crack of concrete structure [8-11]. The concepts of B-scan and C-scan in ultrasonic testing are extended to construct the images of concrete interior using spectral data [12-14]. The spectral B-scan and C-scan can display numerous test results simultaneously. However, if one wants to obtain an overall picture of the crack in the target structure, B-scan or C-scan has to be conducted on several vertical or horizontal test sections. Then, these scan images are used to visualize the location and orientation of the cracks. This is inconvenient and may pose difficulties for the inspectors.

With the progress of computer graphics, 3D display is a trend in processing volume data. The methods that render 3D images are called volume visualization techniques [15-17]. The development of volume visualization was mainly due to the needs in medical practice such as computed tomography (CT), magnetic resonance imaging (MRI), ultrasonic imaging, etc. Recently, the applications of 3D imaging in the non-destructive tests of structures have drawn more and more attention [18-20].

This study applies the surface rendering method, a volume visualization technique, to analyze the impact echo data. The 3D image can be used to determine the location of internal cracks in concrete structures. Both numerical and experimental tests were performed to verify the feasibility and effectiveness of the proposed method.

\section{Imaging methods}

The 3D imaging method is an extension of the 2D imaging methods. Therefore, the spectral B-scan and spectral C-scan will be introduced in the following section.

\subsection{Spectral B-scan and spectral C-scan}

Liu and Yiu [12] proposed the spectral imaging method of the impact echo test based on the B-scan and C-scan of ultrasonic testing. The procedure of spectral B-scan is as follows:

Firstly, one selects a test line on the concrete surface and performs a series of impact echo tests at constant interval along that line. After completing the tests, the FT is applied to the test signals and the amplitude of spectra is represented by color scale

$c= \begin{cases}255 & a>a_{\max } \\ \frac{255 \times\left(a-a_{\min }\right)}{a_{\max }-a_{\min }} & a_{\min }<a<a_{\max } \\ 0 & a<a_{\min }\end{cases}$

where $a$ is the amplitude of the spectrum. $a_{\max }$ and $a_{\min }$, respectively, are the upper and lower bounds of the amplitude, and $c$ is the corresponding color scale. If $a$ exceeds $a_{\max }$, the color scale is set to 255. If $a$ is less than $a_{\mathrm{min}}$, the color scale is set to 0 . One can use $a_{\max }$ and $a_{\min }$ to adjust the contrast of the image. Finally, construct a 2D image using the test position as the horizontal axis and the frequency as the vertical axis. That yields the spectral B-scan of the vertical test section under the test line.

Recall that the amplitude of the Fourier spectrum at each frequency represents the echo energy from a reflector, the depth of which is determined by Eq. (1). If there is no interface on the test section, no obvious color variation should be observed on the image. On the other hand, if a bright zone presents, it may indicate a defect or the bottom of the specimen.

One can extend the same idea to construct the spectral C-scan image of a horizontal cross-section. In the spectral C-scan, one has

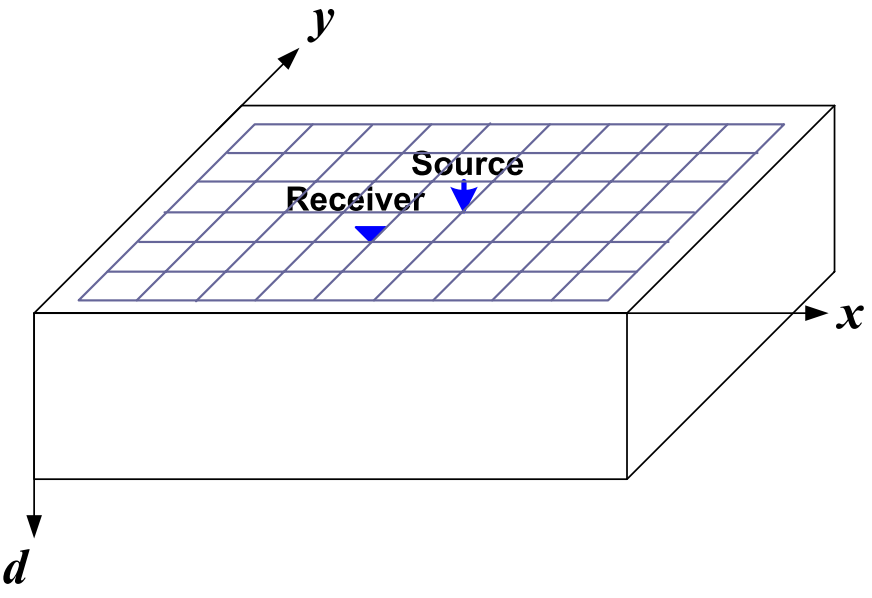

Fig. 1. Test scheme.

to draw a square mesh on the concrete surface and perform the impact echo test at each grid of the mesh, as shown in Fig. 1. Then, the FT is applied to the test signals. If one uses the coordinates of the test positions and the frequency as the axes, one can construct a 3D matrix (or volume data) for the amplitude of the spectra. For a given depth $d$, one can determine the frequency using Eq. (1) and extract the corresponding spectral amplitude from the volume data. Similar to the spectral B-scan, the spectral amplitude is represented by color scale. Finally, using the coordinates of the test positions as the two axes, one can construct a 2D image for the horizontal cross-section, and it is called the spectral C-scan image. Again, a bright zone in the image suggests an interface on the cross-section.

\subsection{The frequency-depth transform}

It should be noted that in the spectral B-scan, the spectra are used directly to construct the image. Hence, it does not provide a "picture" of the test section because the vertical axis is frequency, not depth. The image is easier to interpret if one can transform the frequency into depth. In fact, this transformation is necessary if one wants to extend the 2D imaging method to 3D imaging method.

The transformation of frequency to depth is straightforward. Suppose the frequency resolution of the spectrum is $\Delta f$. One simply applies Eq. (1) to $i \Delta f, i=1,2, \ldots$ to find the corresponding depth $d_{i}$. Then, plotting $a(i \Delta f)$ versus $d_{i}$ yields the depth spectrum of the signal.

The above transformation is suitable if it is applied to transform a single spectrum. It should be modified if the depth spectrum will be used for further image processing. This is because the frequency is inversely proportional to depth. Therefore, a constant interval in $f$ will result in a varying interval in $d$. That is inconvenient for image processing.

An alternative way of transforming frequency to depth is to select a constant $\Delta d$. Apply Eq. (1) to $i \Delta d, i=1,2, \ldots$ to find the corresponding frequency $f_{i}$. Then, plotting $a\left(f_{i}\right)$ versus $i \Delta d$ yields the depth spectrum of the signal. Notice that $f_{i}$ in general is not a multiple of $\Delta f$. Hence, interpolation is needed to find the amplitude at $f_{i}$.

The second approach does provide a spectrum of constant depth interval. However, it is possible that a peak in the original spectrum is omitted because it falls between $f_{i}$ and $f_{i+1}$. The situation is especially severe in the shallow range because the distance between $f_{i}$ and $f_{i+1}$ decreases with $d$. In order to insure that no peak is left out in the depth spectrum, the following modification is suggested. Instead of using $a\left(f_{i}\right)$ as the amplitude 


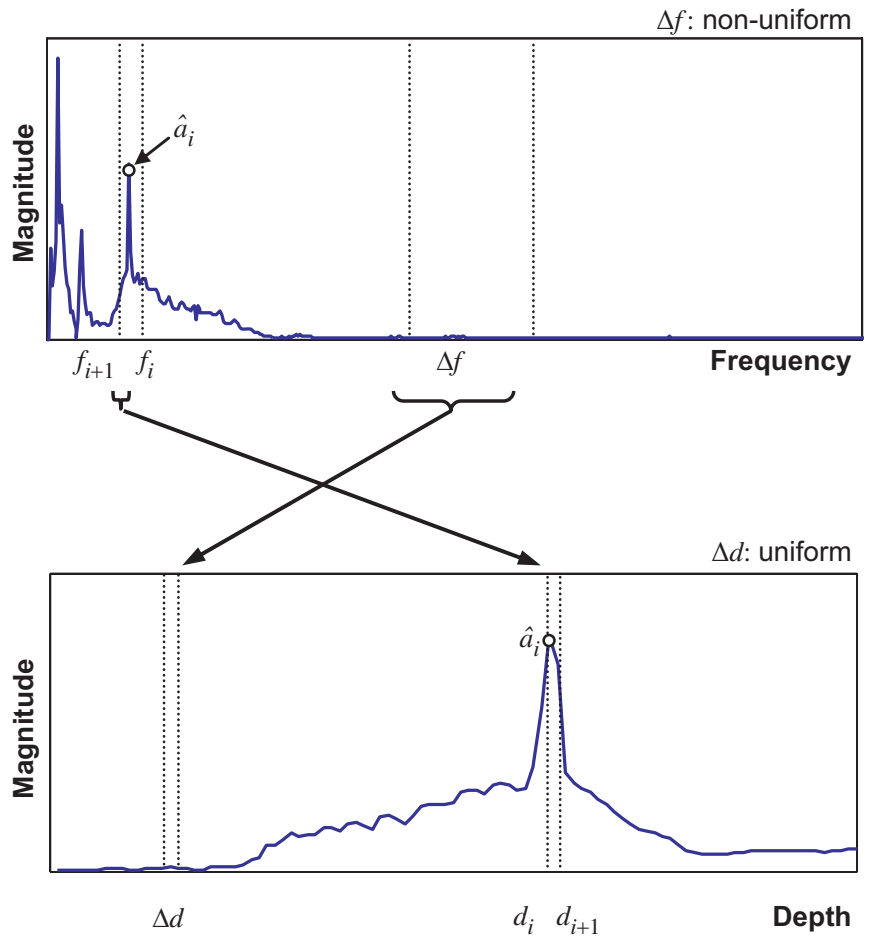

Fig. 2. Frequency-depth transform.

at $i \Delta d$, one determines the maximum amplitude $\hat{a}_{i}$ in the interval $\left(f_{i+1}, f_{i}\right)$, as shown in Fig. 2 . Then, plot $\hat{a}_{i}$ versus $i \Delta d$ to obtain the depth spectrum of the signal.

Using the above transformation, one can obtain a depth spectrum of uniform depth interval, which is suitable for volume visualization.

\subsection{Surface rendering method}

Recall that in spectral C-scan, the impact echo tests are performed at the grids of a square mesh on the concrete surface. Using the coordinates of the test positions and the frequency as the axes, one can construct a 3D matrix for the spectral amplitude. If the depth spectrum is adopted and the frequency axis is replaced by depth axis, the three axes form a conventional $3 \mathrm{D}$ spatial coordinate system. Then, each element in the 3D matrix maps to a voxel in the space. One can use Eq. (3) to find the color scale of each voxel. Such volume data can be used directly in the surface rendering process.

The surface rendering method is equivalent to drawing contour lines in a 2D density plot. Take the B-scan image in Fig. 3a for example. The contour lines of the image corresponding to iso-values 104 and 208 are shown in Fig. 3b. Apparently, if the isovalue is chosen properly, the contour line will depict the location of an interface. The same concept can be extended to the 3D case.

The main idea of surface rendering is to abstract the isosurface from the volume data, i.e., to find a surface with the same color scale. Let $V(x, y, d)$ denote the volume data. The iso-surface corresponding to iso-value $C$ can be represented as

$\{(x, y, d): V(x, y, d)=C\}$

Once an iso-value is assigned, one can use triangular patch to generate an iso-surface [16,21,22]. Similar to the contour lines in Fig. $3 b$, if the iso-value is chosen properly, the iso-surface will depict the location of an interface in the specimen.

After the iso-surface is generated, it is projected to a $2 \mathrm{D}$ view plane. Notice that when a $3 \mathrm{D}$ object is projected to a $2 \mathrm{D}$ plane, a a

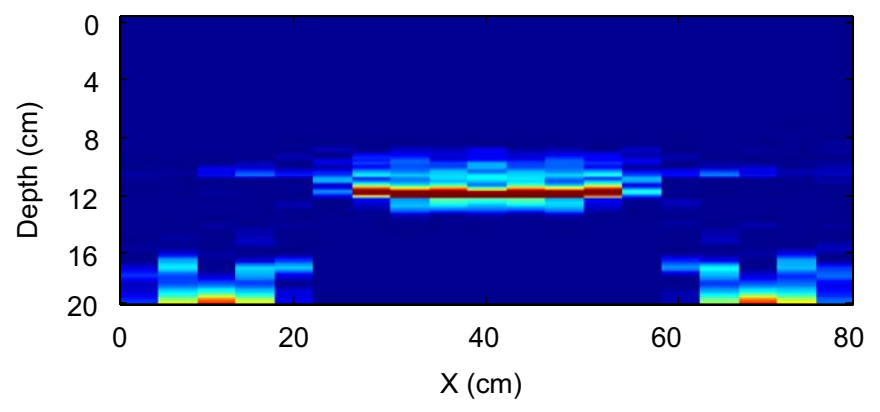

b

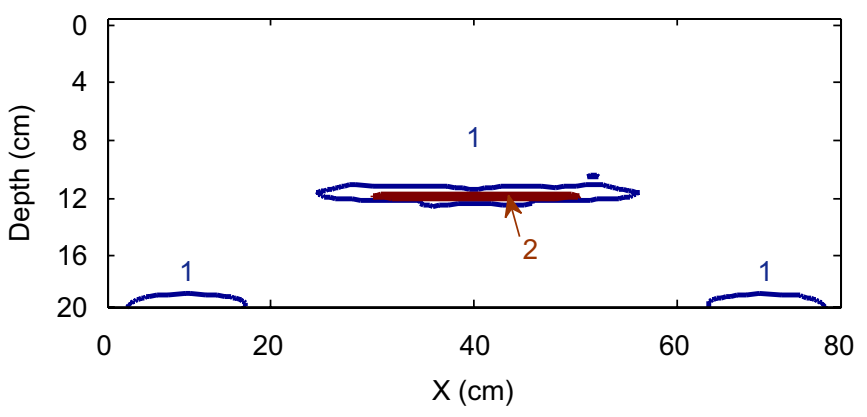

Fig. 3. 2D analogy of surface rendering. (a) B-scan image; (b) Contour plot.

sphere will appear as a circle and a cube is turned into a hexagon. In order to obtain a stereograph, shading and lighting are necessary.

The Phong reflection model is a popular and effective approach to produce a stereograph [16]. This model assumes three types of light-material interactions, namely, ambient, diffuse, and specular reflection. The intensity of the reflected light is dependent on four vectors: the normal vector of the surface $\mathbf{N}$, the viewer vector $\mathbf{V}$, the light source vector $\mathbf{L}$, and the reflected-ray vector $\mathbf{R}$.

The ambient light has uniform intensity in the space. When it encounters a surface, it is absorbed and reflected. The intensity of the ambient reflection $I_{\mathrm{a}}$ is as follows:

$I_{\mathrm{a}}=K_{\mathrm{a}} L_{\mathrm{a}}, \quad 0 \leqslant K_{\mathrm{a}} \leqslant 1$

where $K_{\mathrm{a}}$ is the ambient reflection coefficient and $L_{\mathrm{a}}$ is the intensity of the ambient light.

The diffuse reflection is characterized by the roughness of the surface. The intensity of the reflected light depends on the material and incident direction of the light. Since the normal vector of the surface may vary from point to point, each point reflects different amounts of light. The intensity of the diffuse reflection $I_{\mathrm{d}}$ is as follows:

$I_{\mathrm{d}}=K_{\mathrm{d}} L_{\mathrm{d}} \mathbf{L} \cdot \mathbf{N}, \quad 0 \leqslant K_{\mathrm{d}} \leqslant 1$

where $K_{\mathrm{d}}$ is the diffuse reflection coefficient and $L_{\mathrm{d}}$ is the intensity of the incident diffuse light.

The specular reflection is used to highlight the shiny part of surface. Although the ambient and diffuse reflections make the image look stereoscopic, the lack of specular reflection would make the surface look dull. The intensity of the specular reflection is as follows:

$I_{\mathrm{S}}=K_{\mathrm{s}} L_{\mathrm{S}}(\mathbf{R} \cdot \mathbf{V})^{\alpha}, \quad 0 \leqslant K_{\mathrm{s}} \leqslant 1, \alpha \geqslant 1$

where $K_{\mathrm{s}}$ is the diffuse reflection coefficient, and $L_{\mathrm{s}}$ is the intensity of incident specular light. Usually, $L_{\mathrm{s}}=L_{\mathrm{d}} \cdot \alpha$ is the shininess coefficient. As $\alpha$ increases, the reflected light tends to concentrate on a smaller region. 
The total intensity of the reflected light from an object is the sum of $I_{\mathrm{a}}, I_{\mathrm{d}}$, and $I_{\mathrm{s}}$. In this paper, the coefficients $K_{\mathrm{a}}, K_{\mathrm{d}}, K_{\mathrm{s}}$, and $\alpha$ are taken as $0.6,0.8,0.5$, and 150 , respectively.

In this study, an interactive user interface program is developed. One can rotate the $3 \mathrm{D}$ image of the test specimen arbitrarily so that the interface in the concrete specimen can be easily located.

\section{Numerical simulation}

Two concrete specimens with internal cracks are considered in the numerical examples to illustrate the proposed imaging method. The mass density, Young's modulus, Poisson's ratio, and the longitudinal wave speed of the concrete are $2300 \mathrm{~kg} / \mathrm{m}^{3}$, $33.1 \mathrm{GPa}, 0.2$, and $4000 \mathrm{~m} / \mathrm{s}$, respectively.

The dimensions of concrete specimens are both $80 \mathrm{~cm}(L) \times 80$ $\mathrm{cm}(W) \times 20 \mathrm{~cm}(H)$. A $76 \mathrm{~cm} \times 76 \mathrm{~cm}$ mesh is drawn on the top surface of each specimen, leaving $2 \mathrm{~cm}$ margins on the four sides. The grid lines are drawn at a constant interval of $4 \mathrm{~cm}$ in both directions. Hence, there are totally $19 \times 19$ grids. In each test, the impact source and the receiver are located at the upper right and lower left corners of a grid.

The finite element code LS-Dyna970 [23] was used to simulate the response of concrete specimens due to the impact of a steel ball. Three-dimensional solid elements with side length $1 \mathrm{~cm}$ were used in the numerical models. A time-varying pressure was applied to the surface to simulate the impact of a steel ball with diameter $d=6 \mathrm{~mm}$. According to Goldsmith [24], the pressure can be approximated by a half sine function with a contact time $t_{\mathrm{c}}=0.025 \mathrm{~ms}$. The sampling rate was $344 \mathrm{kHz}$ and the total time of simulation was $3 \mathrm{~ms}$.

\subsection{Numerical model 1}

Numerical model 1 contains a $32 \mathrm{~cm}(L) \times 32 \mathrm{~cm}(W) \times 1 \mathrm{~cm}(H)$ horizontal crack, which is $12 \mathrm{~cm}$ beneath the surface. The coordinate of the center of the crack is $(x, y, d)=(40,40,12)$ (in cm), as shown in Fig. 4.

Fig. 5 shows the oblique, side, and top views of the surface rendering image. Notice that all these images were constructed using the Fourier depth spectra. An iso-surface denoting the crack is observed in all three images at the correct location. One can also find the bottom of the specimen in the image. However, a hole is formed in the bottom beneath the crack, as shown in Fig. 5(a). This is because the bottom echo is blocked by the crack. Therefore, a hole in the bottom gives supplementary evidence for the existence of a defect above this region.

From the side view in Fig. 5(b), one can see that the crack is thicker than the real crack. Actually, the impact echo test cannot reveal the thickness of crack. The thickness of crack in the image

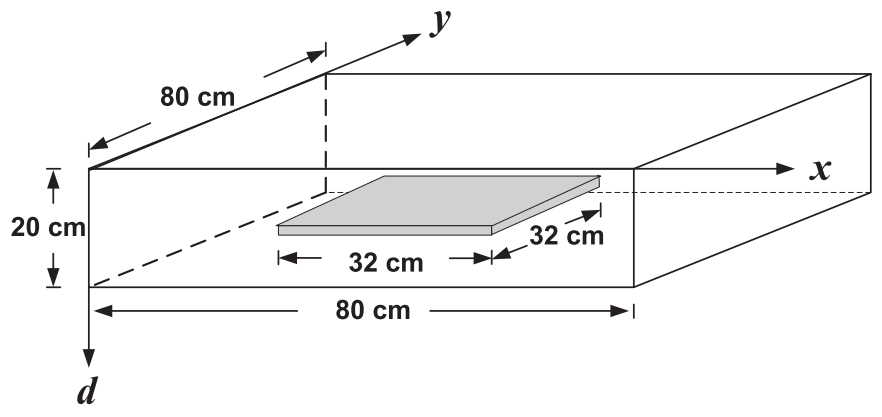

Fig. 4. Test model 1 .
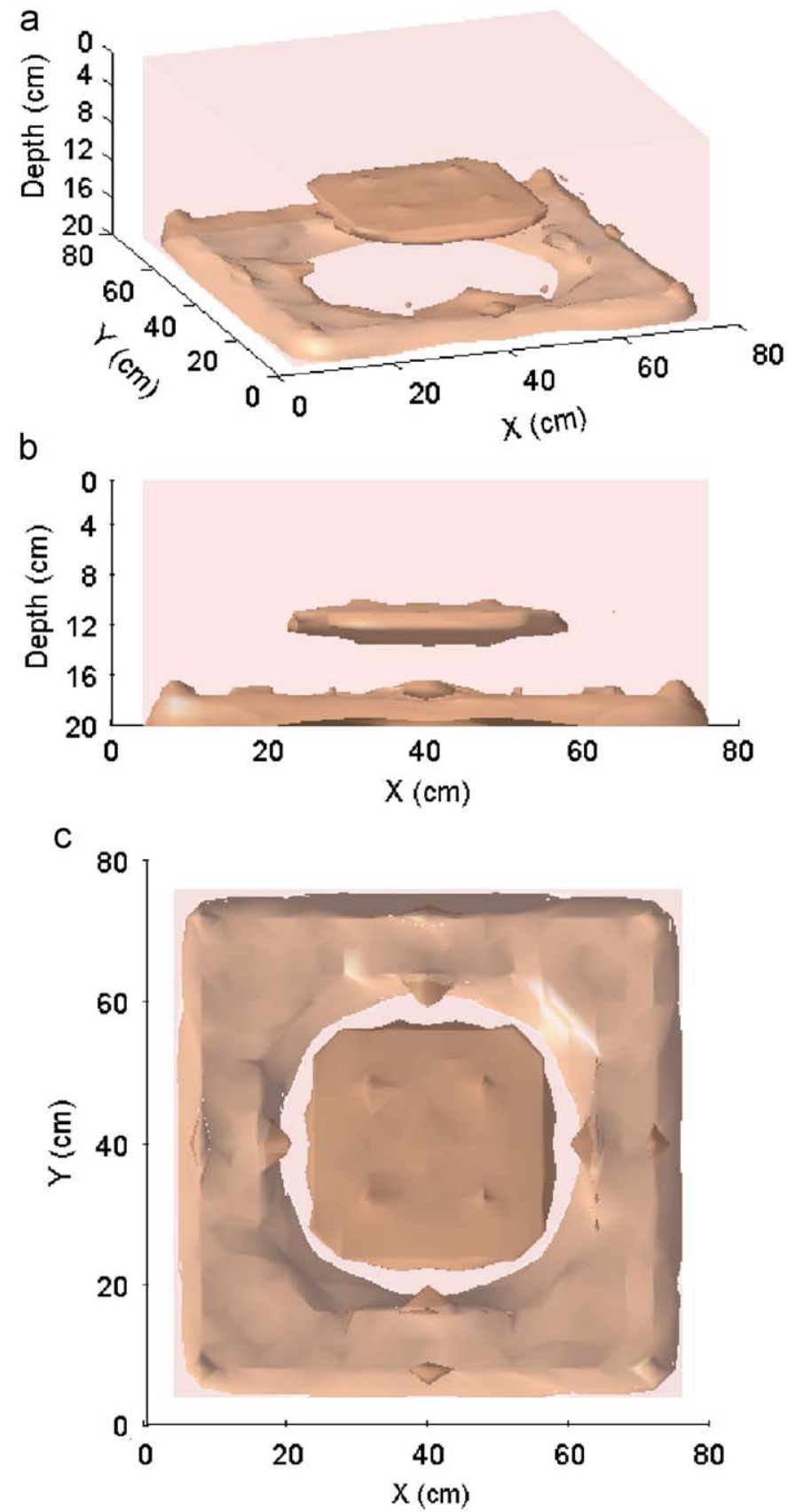

Fig. 5. 3D images of numerical model 1. (a) Oblique view; (b) Side view; (c) Top view.

results from the width of echo peak in the spectra. Therefore, the true depth of the crack is near the center of the iso-surface.

\subsection{Numerical model 2}

Numerical model 2 contains an inclined crack with depth ranging from 6 to $14 \mathrm{~cm}$ beneath the surface. The coordinates of the four corners of the crack are $(x, y, d)=(24,24,14),(24,56,14)$, $(56,24,6)$, and $(56,56,6)$ (in $\mathrm{cm})$, as shown in Fig. 6 .

Fig. 7 shows the oblique, side, and top views of the surface rendering image of model 2. Again, all the images were constructed using the Fourier depth spectra. An iso-surface denoting the crack is observed in all three images. The side view in Fig. 7(b) clearly shows the inclination of the crack. Similar to model 1 , a hole is formed in the bottom beneath the crack. 


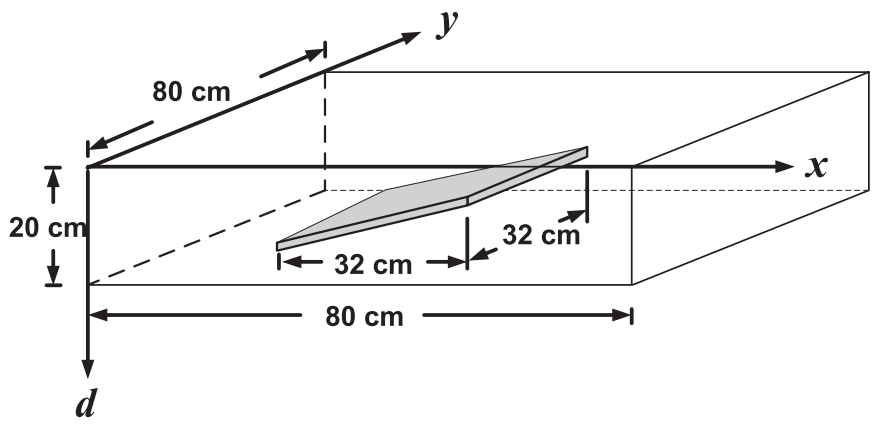

Fig. 6. Test model 2 .

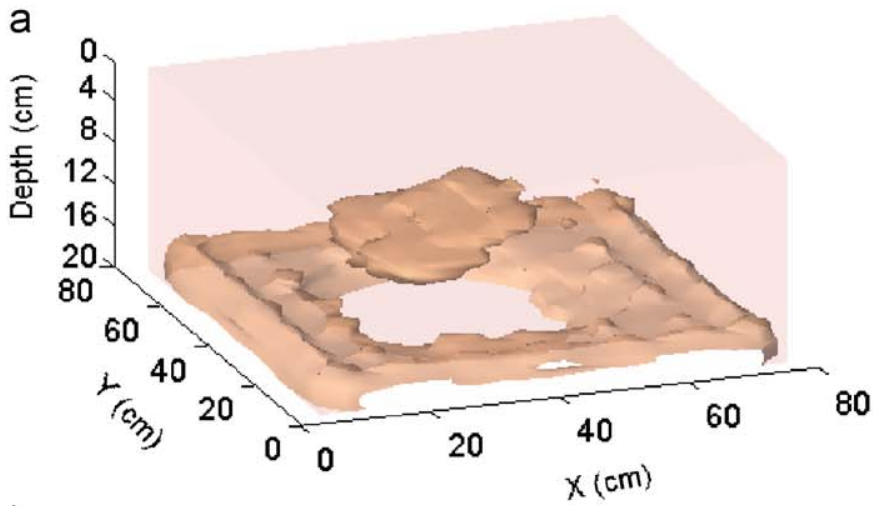

b

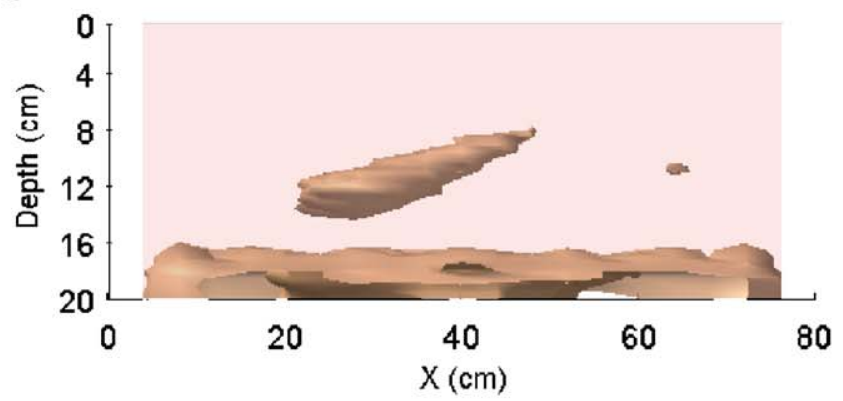

C

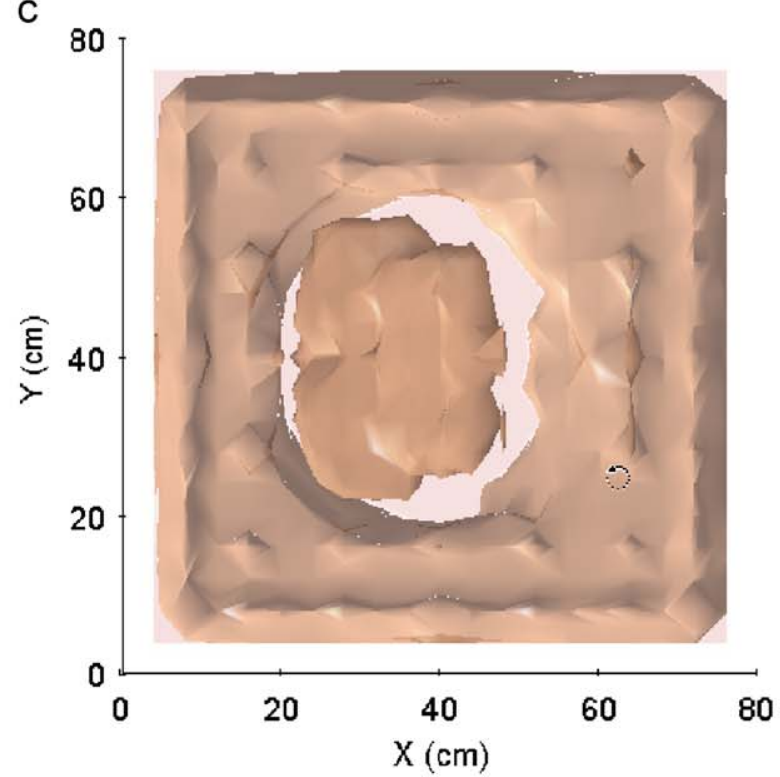

Fig. 7. 3D images of numerical model 2. (a) Oblique view; (b) Side view; (c) Top view.
It is seen that the crack image is narrower and thinner near the shallow edge, and wider and thicker near the deep edge. Furthermore, compared with the true location, the crack image is seen to shift horizontally towards the deep edge. All these phenomena indicate that the amplitude of the echo peaks near the shallow edge is smaller than the amplitude near the deep edge. This is because the same impact source was used throughout the simulation, and the echo energy from different parts of the crack varies. In such case, one may use the hole in the bottom to estimate the size of the crack.

Regardless of the distortion of the crack image, the surface rendering technique provides an overall and clear picture of the interior of the specimen.

\section{Model tests}

Two model tests were conducted in this study to verify the feasibility of the surface rendering method in processing the impact echo data. The dimensions of the concrete specimens and the crack locations are identical to those of the numerical models. The longitudinal wave velocities of models 1 and 2 are 3890 and $3900 \mathrm{~m} / \mathrm{s}$, respectively. Test meshes are also the same as described in the numerical examples.

Unlike the numerical tests, the energy of the impact source may vary from test to test. Therefore, the experimental data need to be normalized. In this study, the test signals are normalized based on the amplitude of the surface wave.

There is another discrepancy between simulation and model test. The experimental data contain a lot of noise. When the Fourier depth spectra were used to construct the 3D image, poor images were rendered. In order to improve the image quality, the enhanced Fourier depth spectra were adopted in the image processing.

\subsection{Model test 1}

The oblique, side, and top views of model 1 are shown in Fig. 8 . Because the experimental signals are contaminated by noise, the 3D images are not as clear as in the numerical examples. The crack is not enveloped in a single iso-surface. Several iso-surfaces appear around the crack location instead. This is mainly because the impact source is unsteady. Normalization of the signals may minimize the influence of the impact intensity, but not the shape. Therefore, it is hard to find an appropriate iso-value to surround the crack by an iso-surface.

Nevertheless, one can still manage to find the crack by viewing the specimen at different angles. For example, in Fig. 8(b) one can detect a horizontal crack around $12 \mathrm{~cm}$ deep; one can also find the bottom surface near the depth of $20 \mathrm{~cm}$. The oblique view in Fig. 8(a) shows a hole in the bottom right beneath the crack. The approximate dimensions of the crack can be obtained from the top view in Fig. 8(c).

It is seen that for experimental data, it is difficult to interpret the image using only a couple of views. The interactive imaging program developed in this study makes the interpretation a lot easier because one may rotate the model freely to obtain a better view of the specimen.

\subsection{Model test 2}

Model 2 contains an inclined crack as shown in Fig. 6. The oblique, side, and top views of this model are shown in Fig. 9. Similar to the first model test, the images contain a lot of noise. However, one can still find several iso-surfaces appearing at the 

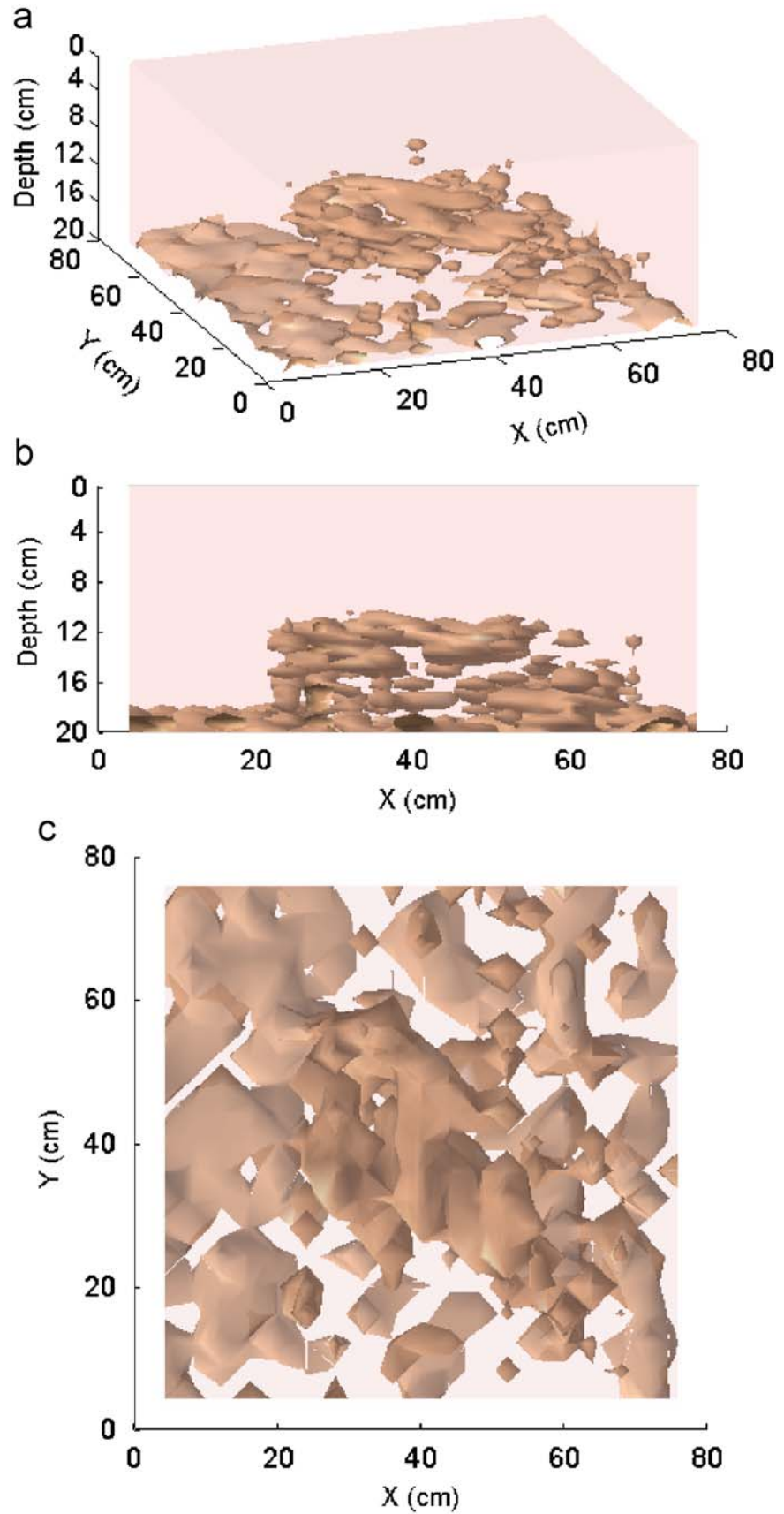

Fig. 8. 3D images of experimental model 1. (a) Oblique view; (b) Side view; (c) Top view.

crack location and the bottom. The hole beneath the crack is still visible.

Although the crack image is formed by several discontinuous iso-surfaces, one can still get an overall picture of the crack. The hole in the bottom can help the inspector to estimate the size of crack.

\section{Conclusion}

This paper applies the surface rendering technique to process the impact echo data so that the 3D image of the internal defects in concrete can be constructed.

Two pre-processing procedure of the impact echo spectra are developed in this study. First, the frequency-depth transform is

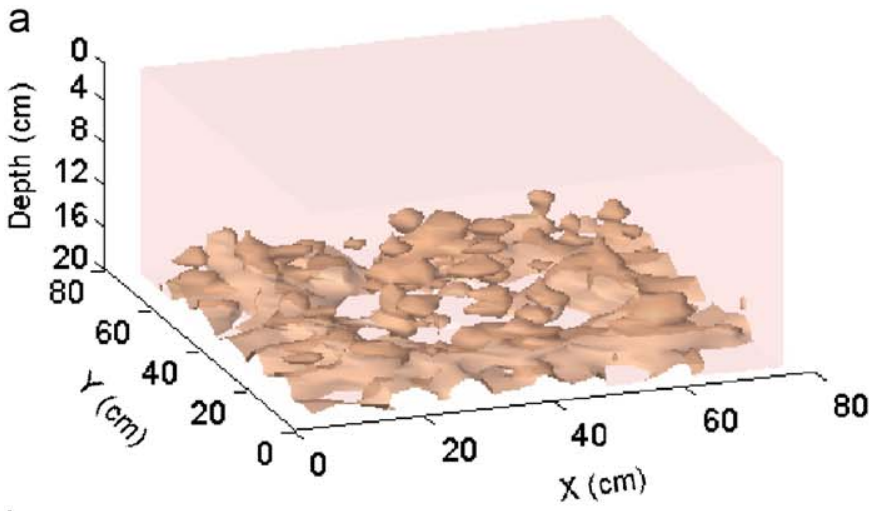

b

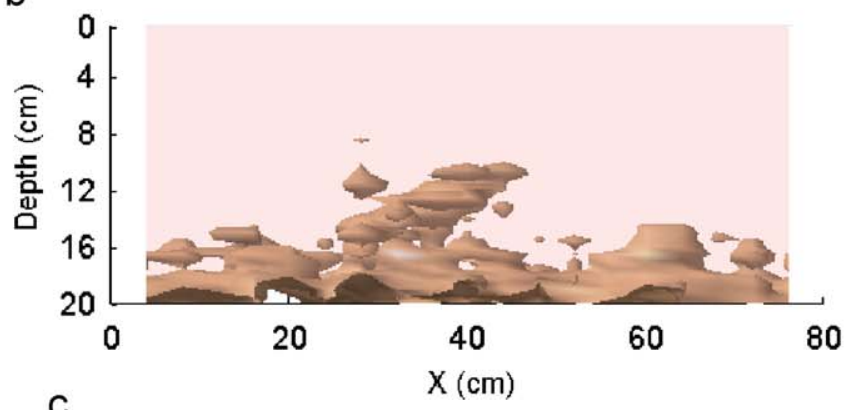

C

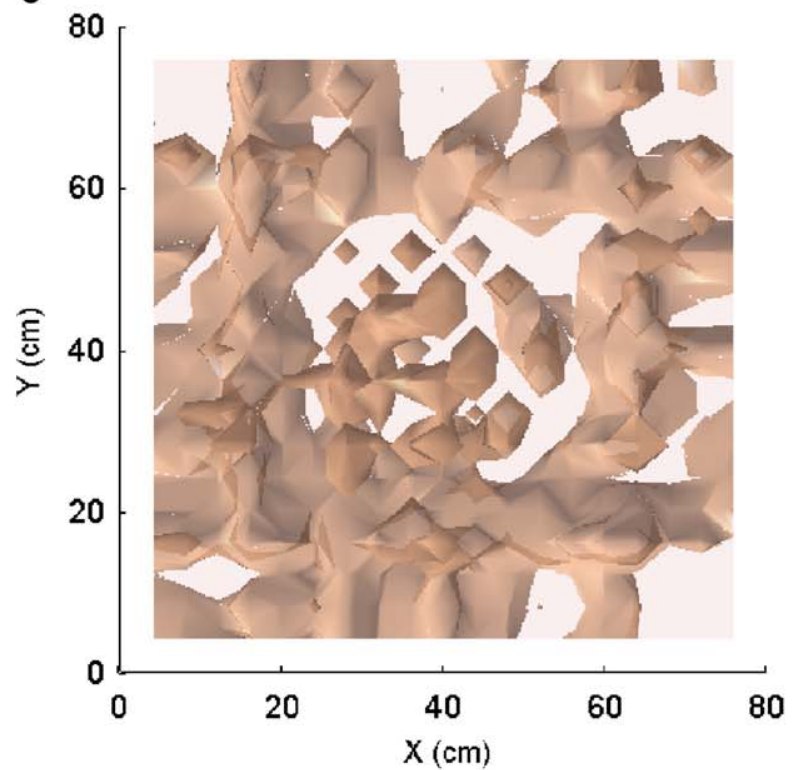

Fig. 9. 3D images of experimental model 2. (a) Oblique view; (b) Side view; (c) Top view.

applied to the Fourier spectra to obtain the depth spectra such that they are suitable for use in 3D imaging. Second, the Fourier spectrum is enhanced by using the wavelet marginal spectrum as a band-pass filter. As such, the feature around the peak is magnified, while the ripples and multiple peaks in the Fourier spectrum are suppressed.

From the numerical examples and model tests, it is shown that the 3D imaging method proposed in this study can depict the interior of the concrete specimen. A crack is represented by one or several iso-surfaces, which can be used to estimate its size, location, and orientation. A hole will appear in the bottom beneath the crack. That provides supplementary information about the size and location of the crack.

Since the experimental data is noisy and the impact source is unsteady, one does not get a clear image of the crack as in the 
numerical examples. In this situation, the interactive imaging program developed in this study provides a useful tool. The inspector may rotate the 3D image arbitrarily to obtain a better view of the crack. Hence, one can still get a rough picture of the concrete interior.

Although the 3D imaging method may provide the most direct information about the defects in concrete structures, its practical applications are hindered by two issues. Firstly, the test is very time-consuming because a vast amount of tests are required. Secondly, the unsteadiness of the impact source deteriorates the quality of the image. To deal with these problems, an automatic test system seems to be a good solution. It is hoped that automatic test systems can be developed in the near future so that the 3D imaging technique can be widely applied in the inspection of concrete structures.

\section{Acknowledgment}

This work was supported by the National Science Council, ROC, under grant NSC 90-2211-E-002-079.

\section{References}

[1] Sansalone M, Carino NJ. Impact-echo: a method for flaw detection in concrete using transient stress waves. Gaithersburg, Maryland: National Bureau of Standards; 1986 (NBSIR 86-3452).

[2] Jeong H, Jang Y-S. Fracture source location in thin plates using the wavelet transform of dispersive waves. IEEE Trans Ultrason, Ferroelectrics, Freq Control 2000;47(3):612-9.

[3] Cook DA, Berthelot YH. Detection of small surface-breaking fatigue cracks in steel using scattering of Rayleigh waves. NDT and E Int 2001;34(7):483-92.

[4] Yan YJ, Yam LH. Detection of delamination damage in composite plates using energy spectrum of structure dynamic responses decomposed by wavelet analysis. Comput Struct 2003;82:347-58.
[5] Darowicki K, Zakowski K. A new time-frequency detection method of stray current field interference on metal structures. Corros Sci 2004;46(5): 1061-70.

[6] Léonard F. Phase spectrogram and frequency spectrogram as new diagnostic tools. Mech Syst Signal Process 2007;21:125-37.

[7] Yeh PL, Liu PL. Application of the wavelet transform and the enhanced Fourier spectrum in the impact echo test. NDT and E Int 2008;41(5):382-94.

[8] Kim YH, Lee S, Kim HC. Quantitative vibration mode analysis of mortar and concrete blocks containing flaws. NDT and E Int 1990;23(2):67-74.

[9] Liu PL, Tsai CD, Wu TT. Imaging of surface-breaking concrete cracks using transient elastic waves. NDT and E Int 1996;29(5):323-31.

[10] Liu PL, Lee KH, Wu TT, Kuo MK. Scan of surface-opening cracks in reinforced concrete using transient elastic wave tests. NDT and E Int 2001;34(3):219-26.

[11] Ohtsu M, Watanabe T. Stack imaging of spectral amplitudes based on impactecho for flaw detection. NDT and E Int 2002;35(3):189-96.

[12] Liu PL, Yiu CY. Imaging of concrete defects using elastic wave tests. In: the 2002 Far-East Conference on Nondestructive Testing. Tokyo, 2002.

[13] Schubert F, Wiggenhauser H, Lausch R. On the accuracy of thickness measurements in impact-echo testing of finite concrete specimensnumerical and experimental results. Ultrasonics 2004;42:897-901.

[14] Yeh PL. The time-frequency domain analysis and image method of the impact-echo method. Taipei: Institute of Applied Mechanics, National Taiwan University, 2006.

[15] Wolff RS, Yaeger L. Visualization of natural phenomena. New York: Springer; 1993.

[16] Angel E. Interactive computer graphics: a top-down approach using OpenGL. 4th ed. Addison Wesley; 2006.

[17] Hearn D, Baker MP. Computer graphics with OpenGL. 3rd ed. Prentice Hall; 2004.

[18] Santos J, Longoria LC, Palacios JC. Detection of flaws in construction columns using 3D reconstruction and manipulation. Linear Algebra Appl 2001;339(1): 195-204.

[19] Kohl C, Krause M, Maierhofer C, Wöstmann J. 2D- and 3D-visualisation of NDT-data using data fusion technique. Mater Struct 2005;38(9):817-26.

[20] Kohl C, Streicher D. Results of reconstructed and fused NDT-data measured in the laboratory and on-site at bridges. Cem Concr Compos 2006;28(4):402-13.

[21] Watt A. 3D computer graphics. 3rd ed. Addison Wesley; 2000.

[22] Udupa JK, Herman GT. 3D imaging in medicine. 2nd ed. Boca Raton: CRC Press; 2000.

[23] Hallquist JO. LS-DYNA keyword user's manual. Livermore: Livermore Software Technology Corporation; 2003.

[24] Goldsmith W. Impact: the theory and physical behavior of colliding solids. London: Edward Arnold Ltd; 1960. 